PROCEEDINGS OF THE

AMERICAN MATHEMATICAL SOCIETY

Volume 51, Number 1, August 1975

\title{
REMARK ON NILPOTENT ORBITS
}

\author{
JOSEPH A. WOLF 1
}

ABSTRACT. If $G$ is a reductive Lie group and $\theta_{f}=\operatorname{Ad}(G)^{*} f$ is a nilpotent coadjoint orbit with invariant real polarization $p$, then $\mathcal{O}_{f}$ is identified as an open $G$-orbit on the cotangent bundle of $G / P$.

Introduction. Let $R^{4,1}$ denote real 5-space with the bilinear form $b(x, y)=x^{1} y^{1}+\ldots+x^{4} y^{4}-x^{5} y^{5}$ and let $\mathcal{C}^{+}$denote its forward light cone $\left\{x \in R^{4,1}: b(x, x)=0\right.$ and $\left.x^{5}>0\right\}$. The rays in $\mathcal{C}^{+}$form a 3 -sphere $S^{3}$, and so the identity component $\mathrm{SO}(4,1)$ of the orthogonal group of $R^{4,1}$ acts on the cotangent bundle $\mathfrak{T}^{*}\left(S^{3}\right)$. This observation is due to $\mathrm{B}$. Kostant, who noted that $\mathrm{SO}(4,1)$ is transitive on the symplectic manifold $\mathcal{T}^{*}\left(S^{3}\right)$-(zero section) and asked Y. Akyildiz to identify that space as a coadjoint orbit for $\mathrm{SO}(4,1)$. Akyildiz identified it as a nilpotent coadjoint orbit, and Kostant noted from dimension considerations that the nilpotent elements in question must be regular-nilpotent. Kostant and I then conjectured that if $G$ is semisimple, $P$ is a minimal parabolic subgroup, and $e \in p$ is a regular-nilpotent element of $g$, then $\operatorname{Ad}(G) \cdot e$ is an open $G$-orbit on the cotangent bundle $\mathcal{T}^{*}(G / P)$. Here note that $\mathrm{SO}(4,1) /($ minimal parabolic $)=$ $\mathrm{SO}(4) / \mathrm{SO}(3)=S^{3}$. The conjecture is proved as Corollary 2 below.

We refer to [1] for the language of polarizations.

Lemma. Let $g$ be a real Lie algebra, $f \in g^{*}$ a linear functional on $g$, and $q \subset g_{C}$ a complex polarization for $f$. If $f(q)=0$ then $q$ is real in the sense $q=p_{C}$ where $p=q \cap g$.

Proof. Let $G$ be a Lie group with Lie algebra $g$ and $E^{0}$ and $D^{0}$ its respective analytic subgroups for

$$
e=(q+\bar{q}) \cap g \text { and } \delta=(q \cap \bar{q}) \cap g \text {. }
$$

$\operatorname{Ad}\left(D^{0}\right)^{*} \cdot f$ is open in the affine subspace $f+e^{\perp}$ of $g^{*}$, and $f \epsilon e^{\perp}$ because $f(q)=0$, so also $\operatorname{Ad}\left(E^{0}\right)^{*} \cdot f$ is open in $f+e^{\perp}$. As $g^{f} \subset \delta \subset e$, now $\operatorname{dim} e=$

Received by the editors May 21, 1974 .

AMS (MOS) subject classifications (1970). Primary 22E45, 53C99; Secondary 22E15, $22 \mathrm{E} 43$.

${ }^{1}$ Research partially supported by NSF Grant GP-16651. 
$\operatorname{dim}\left(f+e^{\perp}\right)-\operatorname{dim} g^{f}=\operatorname{dim} \delta$, this forces $e=\delta$, and we conclude $q=\bar{q}_{\bullet} \quad$ Q.E.D.

Theorem. 2 Let $G$ be a Lie group, $f \in \mathrm{g}^{*}$, q a complex polarization for $f$ such that $f(q)=0$, and $p=q \cap g$. Let $P$ be a closed subgroup of $G$ with Lie algebra $p$ such that $G^{f} \subset P$. Then $\Theta_{f}=\operatorname{Ad}(G)^{*} \cdot f$ is equivariantly diffeomorphic to an open $G$-orbit in the cotangent bundle $\mathcal{T}^{*}(G / P)$. of $g^{*}$.

Proof. As in the lemma, $\operatorname{Ad}(P)^{*} \cdot f$ is open in the subspace $f+p^{\perp}=p^{\perp}$

$G / P$ has tangent space $g / p$, hence cotangent space $(g / p)^{*}=p^{\perp}$, all this as $P$-modules. Thus $\mathcal{J}^{*}(G / P)$ is the $G$-homogeneous bundle $G \times_{P} F^{\perp}$. It consists of all classes

$$
[g, y]=\left\{\left(g p^{-1}, \operatorname{Ad}(p)^{*} y\right): p \in P\right\} \subset G \times p^{\perp}
$$

with quotient differentiable structure from $G \times p^{\perp}$ and with left action of $G$ given by $g^{\prime}[g, y]=\left[g^{\prime} g, y\right]$. Define a $G$-orbit on $\mathcal{T}^{*}(G / P)$ by

$$
\Omega_{f}=G([1, f])=\left\{[g, f] \in G \times_{P} P^{\perp}: g \in G\right\} .
$$

Then

$$
\begin{aligned}
\operatorname{dim} \Omega_{f} & =\operatorname{dim}(G / P)+\operatorname{dim}\left(\operatorname{Ad}(P)^{*} \cdot f\right)=\operatorname{dim} g-\operatorname{dim} \not+\operatorname{dim} p^{\perp} \\
& =\operatorname{dim}\left(G \times_{P} p^{\perp}\right)=\operatorname{dim} \mathcal{T}^{*}(G / P),
\end{aligned}
$$

so $\Omega_{f}$ is open in $\mathcal{T}^{*}(G / P)$.

Map the orbit $\mathcal{O}_{f}$ to $\Omega_{f}$ by $\operatorname{Ad}(g)^{*} f \mapsto[g, f]$. This is well defined, for if $\operatorname{Ad}(g)^{*} f=\operatorname{Ad}\left(g^{\prime}\right)^{*} f$ then $g^{\prime}=g p$ with $p \in G^{f} \subset P$ so $\left[g^{\prime}, f\right]=[g p, f]=$ $\left[g, A d(p)^{*} f\right]=[g, f]$. It is visibly $G$-equivariant with image $\Omega_{f}$, and is oneto-one because $[g, f]=\left[g^{\prime}, f\right]$ forces $\left[g^{-1} g^{\prime}, f\right]=[1, f]$ whence $g^{-1} g^{\prime} \epsilon$ $G^{f} \subset P$. Q.E.D.

We' now suppose that $G$ is a reductive Lie group, i.e. that its Lie algebra $g=g_{1} \oplus c$ where $c$ is the center and $g_{1}=[g, g]$ is semisimple. We also suppose that $g$ has a nondegenerate $\operatorname{Ad}(G)$-invariant symmetric bilinear form $\langle$,$\rangle . That is automatic for example if \left\{\left.A \mathrm{~d}(g)\right|_{c}: g \in G\right\}$ is precompact, e.g. when if $g \in G$ then $\operatorname{Ad}(g)$ is an inner automorphism on $g_{C}$, in particular when $G$ is connected. The form $\langle$,$\rangle gives a G$-equivariant isomorphism of $g$ to $g^{*}$, say $x \rightarrow x^{*}$, by $x^{*}(y)=\langle x, y\rangle$. We say that $x$ and $x^{*}$,

2 Originally we started with Corollary 1 below (same proof). Alan Weinstein suggested the possibility of a more general formulation. 
and their G-orbits, are "nilpotent" when $x \in[g, g]$ with ad( $x): g \rightarrow g$ nilpotent as linear transformation.

Combining [2, Theorem 2.2] and [3, Proposition 2.3.2] we have

Lemma. Let $G$ be reductive as above, $x \in g$, and $q$ a complex polarization for $x^{*}$. Then $q$ is a parabolic subalgebra of $g_{C}$, and $x^{*}(q)=0$ if and only if $x$ is nilpotent.

Now we can prove

Corollary 1. Let $G$ be reductive as above, $e \in g$ a nilpotent element, $q$ a complex polarization for $e^{*}$, and $P$ the parabolic subgroup of $G$ with Lie algebra $p=q \cap g$. Then $\operatorname{Ad}(G) \cdot e$ is equivariantly diffeomorphic to an open $G$-orbit on $\mathcal{J}^{*}(G / P)$ if, and only if, the polarization $q$ is $\operatorname{Ad}\left(G^{e}\right)$ invariant.

Proof. If $q$ is $\operatorname{Ad}\left(G^{e}\right)$-invariant, then $G^{e} \subset P$, and the Theorem realizes $\operatorname{Ad}(G) \cdot e$ as an open $G$-orbit on $\mathcal{T}^{*}(G / P)$. If $\operatorname{Ad}(G) \cdot e$ is equivariantly diffeomorphic to an open $G$-orbit on $\mathcal{T}^{*}(G / P)$, then the diffeomorphism must be given as in the proof of the Theorem; that requires $G^{e} \subset P$, and so $q$ is $\operatorname{Ad}\left(G^{e}\right)$-invariant. Q.E.D.

If $e \in g$ is regular-nilpotent then $e$ is contained in a unique minimal parabolic subalgebra $p$ of $g$. Now $e$ is in the nilradical $p_{n}=p^{\perp}$, so $q=p_{C}$ is a complex polarization for $e^{*}$, and $q$ is $\operatorname{Ad}\left(G^{e}\right)$-invariant by uniqueness of $p$. Thus Corollary 1 specializes to

Corollary 2. Let $G$ be reductive as above, $e \in g$ a regular-nilpotent element, and $P$ the unique minimal parabolic subgroup of $G$ whose Lie algebra contains $e$. Then $\operatorname{Ad}(G) \cdot e$ is G-equivariantly diffeomorphic to an open G-orbit on $\mathcal{J}^{*}(G / P)$.

Remarks. 1. When $[g, g]$ is isomorphic to the Lie algebra of $\operatorname{SO}(n, 1)$, then in Corollary 2 we have $A \mathrm{~d}(P) \cdot e=p_{n}-\{0\}=p^{\perp}-\{0\}$, so the open $G$-orbit is $\mathcal{T}^{*}(G / P)-($ the zero cross section).

2. Let $P$ be any parabolic subgroup of $G$, $P$ its Lie algebra, and $p_{n}$ the nilradical of t. R. W. Richardson and C. C. Moore independently showed that there are open $\operatorname{Ad}(P)$-orbits on $p_{n}$. If $\operatorname{Ad}(P) \cdot e$ is one of them, then ${ }^{3}$ $e^{*}(p)=0$, and a dimension count shows that $q=p_{C}$ is a complex polarization for $e^{*}$. Conversely if $e \in g$ is nilpotent and $q$ is a complex polarization

3 This was pointed out to me by B. Kostant. 
for $e^{*}$, then our lemmas show $q=\mathfrak{p}_{C}$ with $\not p$ parabolic in $g, e \in p^{\perp}=p_{n}$ and $\operatorname{Ad}(P) \cdot e$ open in $P_{n}$. But there are many instances in which $q$ is not $\operatorname{Ad}\left(G^{e}\right)$-invariant. The invariant case is characterized in Corollary 1.

\section{REFERENCES}

1. L. Auslander and B. Kostant, Polarization and unitary representations of solvable Lie groups, Invent. Math. 14 (1971), 255-354. MR 45 \#2092.

2. H. Ozeki and M. Wakimoto, On polarizations of certain homogeneous spaces, Proc. Japan Acad. 48 (1972), 1-4. MR 47 \#402.

3. L. P. Rothschild and J. A. Wolf, Representations of semisimple groups as sociated to nilpotent orbits, Ann. Sci. École Norm. Sup. 7 (1974), 155-174.

DEPARTMENT OF MATHEMATICS, UNIVERSITY OF CALIFORNIA, BERKELEY, CALIF ORNIA 94720

Current address: Institute of Mathematics, The Hebrew University, Jerusalem, Israel 